Working

Paper

Department

of Economics

$\mathrm{Ca}^{\prime}$ Foscari University of

Venice

Guido Cazzavillan

Patrick Pintus

Dynamic Inefficiency in an

Overlapping Generations

Economy with Production 


\title{
Dynamic Inefficiency \\ in an Overlapping Generations Economy with Production
}

\author{
Guido Cazzavillan \\ University of Venice and SET, Italy
}

\section{Patrick Pintus}

Université de la Méditerranée and GREQAM-IDEP, France

\begin{abstract}
Reichlin (JET, 1986) has shown in an OLG model with productive capital that whenever the steady state is locally indeterminate and undergoes a Hopf bifurcation, it is Pareto-optimal. While these results were established under the assumption of Leontief technology, the author has partially extended them to show that the Hopf bifurcation is robust with respect to the introduction of capitallabor substitution. In this note, we prove that the Pareto-optimality of the steady state does not extend to technologies with capital-labor substitution. When the steady state is a sink or undergoes a Hopf bifurcation, it is characterized by over-accumulation with respect to the Golden Rule - the interest rate is negative - hence not Pareto-optimal. Most importantly, it follows that stabilization policies targeting the steady state leave room for welfare losses associated with productive inefficiency, apart from the very special case of Leontief technology.
\end{abstract}

\section{Keywords}

Endogenous fluctuations, local bifurcations, dynamic inefficiency.

\section{JEL Codes}

C62, D61, E32.

\author{
Address for correspondence: \\ Guido Cazzavillan \\ Department of Economics \\ Ca' Foscari University of Venice \\ Cannaregio 873, Fondamenta S.Giobbe \\ 30121 Venezia - Italy \\ Phone: (++39) 0412349152 \\ Fax: (++39) 0412349176 \\ e-mail: guido@unive.it
}

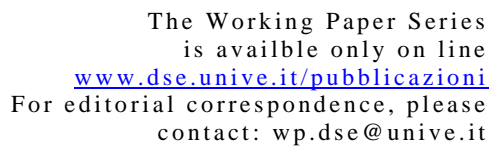

The Working Paper Series is availble only on line www.dse.unive.it/pubblicazion For editorial correspondence, please contact:wp.dse@unive.it

Department of Economics

Ca' Foscari University of Venice

Cannaregio 873, Fondamenta San Giobbe

30121 Venice Italy

Fax: ++390412349210 


\section{Introduction $^{1}$}

In an influential article that appeared in a special issue published by the Journal of Economic Theory (vol. 40, no. 1, october 1986), Reichlin [4] has shown that Hopf cycles arise in overlapping generations economies under gross substitutability, i.e. when the amount saved by the young generation is an increasing function of the interest rate. Reichlin $[4, \mathrm{p}$. 90] states in the introduction that "a particular feature of the present model is that the unique steady state arising from the set of competitive equilibrium trajectories is also the unique Pareto-optimal allocation [...]". In fact, the purpose of the appendix in Reichlin [4, pp. 100-1] is to show that, under Leontief technology, the stationary allocation is Pareto-optimal. Reichlin [4, Section 4] has partially extended the analysis to show that the occurrence of Hopf cycles is robust with respect to the introduction of capital-labor substitution.

In this note, we complete Reichlin's analysis by asking whether the

\footnotetext{
${ }^{1}$ The authors thank Jean-Michel Grandmont and an anonymous associate editor for very helpful suggestions. However, we are especially indebted to Pietro Reichlin for his comments on the first draft which helped us to improve the content of this note and for pointing out a mistake that was contained in an earlier version.
} 
steady state Pareto-optimality extends to technologies with capital-labor substitution, such as those considered in Reichlin [4, Section 4]. Our answer is negative, as we prove that the stationary allocation is not Pareto-optimal (see Proposition 2.1), in contrast with the case of Leontief technology studied in Reichlin's [4]. More precisely, when technology is close to the Leontief case, in the sense that the elasticity of input substitution is arbitrarily close to (but different from) zero, the interest rate is negative so that dynamic inefficiency prevails. As a consequence, the steady state is not Pareto-optimal when it is a sink or when it undergoes a Hopf bifurcation.

The fact that the steady state is, for a broad class of technologies, typically not Pareto-optimal has nontrivial implications for the kind of stabilization policies studied in Reichlin's [4, Section 3]. In view of our results stated in Propositions 2.1, Hopf cycles surround a dynamically inefficient steady state so that policies targeting the stationary allocation leave room for welfare losses associated with productive inefficiency, except in the very special case of Leontief technology.

It should be emphasized that the results cannot be inferred from Cazzavillan and Pintus [3, Proposition 3.3], as we consider there a setting which, unlike Reichlin's, allows for consumption by the young generation 
and there seems to be no way to show that there exists a smooth transition from the latter setting to that with no consumption in the first period of life.

\section{Results}

In the competitive, non-monetary economy studied in this section (see Reichlin [4]), a unique good is produced in the economy by combining labor $l_{t} \geq 0$ and the capital stock $k_{t-1} \geq 0$ resulting from the previous period. Production exhibits constant returns to scale, so that output is given by:

$$
F(k, l) \equiv \operatorname{Alf}(a)
$$

where $A>0$ is a scaling parameter and the latter equality defines the production function defined upon the capital labor ratio $a=k / l$. On technology, we assume the following. 


\section{Assumption 2.1}

The production function $f(a)$ is continuous for $a \geq 0, C^{r}$ for $a>0$ and $r$ large enough, with $f^{\prime}(a)>0$ and $f^{\prime \prime}(a)<0$.

Notice that the technology considered in Assumption 2.1 nests, as a particular case, the CES class studied in Reichlin [4, Section 4]. Competitive firms take real rental prices of capital and labor as given and determine their input demands by equating the private marginal productivity of each input to its real price. Accordingly, the real competitive equilibrium wage is:

$$
\omega=\omega(a) \equiv A\left[f(a)-a f^{\prime}(a)\right]
$$

while the real competitive gross return on capital is:

$$
R=R(a) \equiv A f^{\prime}(a)+1-\delta,
$$

where $1 \geq \delta \geq 0$ is the constant depreciation rate for capital. The produced good can be either consumed or saved as investment by a constant population of households living two periods. Agents are identical within each generation, supply labor and save their wage income in the form of capital when young, to be consumed when old. Agents born at time $t$ 
solve the following program:

$\max \left\{V_{2}\left(c_{t+1} / B\right)-V_{1}\left(l_{t}\right)\right\}$ such that $k_{t}=\omega_{t} l_{t}, c_{t+1}=R_{t+1} k_{t}, c_{t+1} \geq 0, l_{t} \geq 0$.

where $B>0$ is a scaling parameter, $c_{t+1}$ is next period consumption, $l_{t}$ is labor supply, $R_{t+1}>0$ is next period gross return on capital (assumed to be perfectly foreseen). We consider the case such that leisure and consumption are gross substitutes and assume therefore the following:

\section{Assumption 2.2}

The utility functions $V_{1}(l)$ and $V_{2}(c)$ are continuous for $l^{*} \geq l \geq 0$ and $c \geq 0$, where $l^{*}>0$ is the (maybe infinite) workers' endowment of labor. They are $C^{r}$ for, respectively, $0<l<l^{*}$ and $c>0$, and $r$ large enough, with $V_{1}^{\prime}(l)>0, V_{1}^{\prime \prime}(l)>0, \lim _{l \rightarrow l^{*}} V_{1}^{\prime}(l)=+\infty$, and $V_{2}^{\prime}(c)>0$, $V_{2}^{\prime \prime}(c)<0,-c V_{2}^{\prime \prime}(c)<V_{2}^{\prime}(c)$ (that is, consumption and leisure are gross substitutes).

Under Assumption 2.2, it is easily shown that the first-order conditions of problem (4) are:

$$
v_{2}\left(c_{t+1}\right)=v_{1}\left(l_{t}\right) \text { and } c_{t+1}=R_{t+1} \omega_{t} l_{t},
$$


where $v_{1}(l) \equiv l V_{1}^{\prime}(l)$ and $v_{2}(c) \equiv c V_{2}^{\prime}(c / B) / B$. Therefore, intertemporal equilibria may be summarized by the dynamics of both $a$ and $k$, as follows.

\section{Definition 2.1}

An intertemporal perfectly competitive equilibrium with perfect foresight is a sequence $\left(a_{t}, k_{t-1}\right)$ of $\mathbf{R}_{++}^{2}, t=1,2, \ldots$, such that

$$
\left\{\begin{aligned}
v_{2}\left(R\left(a_{t+1}\right) k_{t}\right) & =v_{1}\left(k_{t-1} / a_{t}\right), \\
k_{t} & =\omega\left(a_{t}\right) k_{t-1} / a_{t} .
\end{aligned}\right.
$$

In an appendix, Reichlin [4, pp. 100-1] shows that, under Leontief technology, the stationary allocation is Pareto-optimal. By considering a broader class of technologies with capital-labor substitution, we now prove that this result does not hold when the elasticity of input substitution is positive: the interest rate is negative when the steady state of equations (6) is a sink or undergoes a Hopf bifurcation, that is, dynamic inefficiency prevails. In such a case, Pareto-optimality fails to hold, in contrast to what happens under Leontief technology. In other words, if one leaves the fixed-coefficient production function, the reversal of the Pareto-optimality property of the stationary solution always holds. 


\section{Lemma 2.1 (Dynamic Inefficiency Under Capital-Labor Substitution)}

Assume that capital and labor are not complementary in production, i.e. that the elasticity of input substitution is positive. Then whenever the steady state of equations (6) is a sink or undergoes a Hopf bifurcation, the gross return to capital is less than one or, equivalently, the interest rate is negative.

Proof: The existence of an interior steady state $(\bar{a}, \bar{k})>0$ of equations (6) can be established by choosing the scaling parameters $A$ and $B$ and imposing some boundary conditions on the production and utility functions. This procedure ensures the persistence of the steady state when parameters are varied. ${ }^{2}$ Now letting $\varepsilon_{R}, \varepsilon_{\omega}, \varepsilon_{\gamma}$ be the elasticities of the functions $R(a), \omega(a), \gamma(l)$, respectively, evaluated at the steady state $(\bar{a}, \bar{k})$, one can show that $\varepsilon_{\omega}=s / \sigma$ and $\varepsilon_{R}=-\theta(1-s) / \sigma$, where $\theta \equiv s /[1-\delta(1-s)]$ and $\sigma \equiv d \ln (k / l) / d \ln (\omega /[R-1+\delta])$ is the elasticity of input substitution. Moreover, Assumption 2.2 implies that $v_{1}$ and $v_{2}$ are increasing while $v_{1}$ is onto $\mathbf{R}_{+}$so that one can define the function $\gamma(l) \equiv v_{2}^{-1} \circ v_{1}(l)$, whose graph is the offer curve, with $\varepsilon_{\gamma}>1$.

\footnotetext{
${ }^{2}$ See Cazzavillan et al. [1] and Cazzavillan [2].
} 
The Jacobian matrix of equations (6), evaluated at the steady state $(\bar{a}, \bar{k})>0$, has determinant $D$ which is equal to the following expression:

$$
D=\varepsilon_{\gamma} \varepsilon_{\omega} /\left|\varepsilon_{R}\right|>0
$$

A necessary condition for local indeterminacy (when the steady state is a sink) and Hopf bifurcation is $1 \geq D$ or, as $\varepsilon_{\gamma}>1, \varepsilon_{\omega} /\left|\varepsilon_{R}\right|<1$. From equations (7) and the definitions of elasticities, one gets that $\varepsilon_{\omega} /\left|\varepsilon_{R}\right|=$ $[1-\delta(1-s)] /(1-s)$. Moreover, using that $R(\bar{a})=A f^{\prime}(\bar{a})+1-\delta$ with $R(\bar{a}) \bar{k}=\bar{c}=A f(\bar{a})-\delta \bar{k}$, and defining $s=\bar{a} f^{\prime}(\bar{a}) / f(\bar{a})$ as the share of output going to capital, one has that $R(\bar{a})=s A f(\bar{a}) / \bar{a}+1-\delta=s(R(\bar{a})+$ $\delta)+1-\delta$. Solving the latter equality yields $R(\bar{a})=[1-\delta(1-s)] /(1-s)=$ $\varepsilon_{\omega} /\left|\varepsilon_{R}\right|$ (or, under full depreciation as in Reichlin [4], $R(\bar{a})=s /(1-s)$ ). In summary, local indeterminacy and Hopf bifurcation implies $R(\bar{a})<1$ (or $s<\delta(1-s)$ ), which proves our claim that the interest $r(\bar{a})=R(\bar{a})-1$ is then negative at steady state.

Lemma 2.1 proves that dynamic inefficiency of the steady state follows from local indeterminacy or Hopf bifurcation. We now show that, consequently, the steady state is not Pareto-optimal under capital-labor substitution, i.e. when the elasticity of input substitution is positive. 


\section{Proposition 2.1 (Pareto-Dominated Steady State)}

Under the assumptions of Lemma 2.1, the steady state is always not Pareto optimal, as there always exist some other allocations that produce higher consumption for some periods and never less consumption.

Proof: Assume that the economy is at steady state and let $\bar{k}$ and $\bar{l}=\bar{k} / \bar{a}$ denote the steady-state values of capital and labor. We now show that reducing in period $t_{0}$ the capital stock by $\varepsilon>0$ improves consumption at all dates. Then consumption at $t_{0}$ is increased by $\varepsilon>0$ while consumption at all future dates $t>t_{0}$ is given by $C(\bar{k}-\varepsilon, \bar{l}) \equiv F(\bar{k}-\varepsilon, \bar{l})-\delta(\bar{k}-\varepsilon)$. By concavity, one has that $F(k-\varepsilon, l) \geq F(k, l)-\varepsilon F_{k}(k-\varepsilon, l)$. Therefore, it follows that:

$$
\begin{aligned}
C(\bar{k}-\varepsilon, \bar{l}) & \geq F(\bar{k}, \bar{l})-\varepsilon F_{I}(\bar{k}-\varepsilon, \bar{l})-\delta(\bar{k}-\varepsilon) \\
& \geq C(\bar{k}, \bar{l})+\varepsilon\left(\delta-F_{k}(\bar{k}-\varepsilon, \bar{l})\right) \\
& >C(\bar{k}, \bar{l}),
\end{aligned}
$$

where the last inequality follows from the fact that $R(\bar{a})<1$ holds at steady state, as Lemma 2.1 above has shown. More precisely, $R(\bar{a})=$ $F_{k}(\bar{k}, \bar{l})+1-\delta<1$ so that $F_{k}(\bar{k}-\varepsilon, \bar{l})<\delta$ for some small enough $\varepsilon$. As a consequence, consumption is increased for all generations, which generates a higher welfare. 
So as to understand why Pareto-optimality, while holding under Leontief technology, fails under input substitution, we now restate the proof of Pareto-optimality under capital-labor complementarity, in an arguably simpler way compared to Reichlin's [4] appendix.

\section{Proposition 2.2 (Pareto-Optimal Steady State)}

Assume that capital and labor are complementary in production, i.e. that the elasticity of input substitution is zero. Then steady state is Pareto optimal.

Proof: Using Reichlin's [4, p. 101] notations, define $W_{0}=V_{2}\left(\left[\left(1 / a_{1}+1-\right.\right.\right.$ $\left.\left.\delta) k_{0}-k_{1}\right] / B\right)$ and $W_{t}=V_{2}\left(\left[\left(1 / a_{1}+1-\delta\right) k_{t}-k_{t+1}\right] / B\right)-V_{1}\left(a_{0} k_{t-1} / a_{1}\right)$ as the welfare of generation 0 and $t \geq 1$, respectively, where $a_{1}$ and $a_{0}$ denote the capital-output and labor-output ratios (that is, $k_{t-1} / l_{t}=a_{1} / a_{0}$ ). Defining the capital stock and welfare at the steady state by, respectively, $\bar{k}$ and $\overline{W_{i}}$, where $i=y, o$ for the young and the old generations, respectively, we set $k_{0}=\bar{k}, k_{1}=\bar{k}-\varepsilon$, for some $\varepsilon>0$. Then $W_{0}=V_{2}\left(\left[\left(1 / a_{1}-\delta\right) \bar{k}+\varepsilon\right] / B\right)>\overline{W_{o}}=V_{2}\left(\left[\left(1 / a_{1}-\delta\right) \bar{k}\right] / B\right)$ clearly holds for the old generation. We now show that $W_{t}<\overline{W_{y}}$ for all $t \geq 1$. By 
definition, $\overline{W_{y}}=V_{2}\left(\left[\left(1 / a_{1}-\delta\right) \bar{k}\right] / B\right)-V_{1}\left(a_{0} \bar{k} / a_{1}\right)$ for the young generation. It is easy to check that $\overline{W_{y}}$ is a strictly concave function of $\bar{k}$ (because of utility concavity) and that it has a global maximum for $k=\bar{k}$. To see this, compute $d \overline{W_{y}} / d \bar{k}=\left(1 / a_{1}-\delta\right) V_{2}^{\prime}(\bar{c} / B)-a_{0} V_{1}^{\prime}(\bar{l}) / a_{1}$. Using that $\bar{c}=\left(1 / a_{1}-\delta\right) \bar{k}$ and $\bar{l}=a_{0} \bar{k} / a_{1}$, one further shows that $d \overline{W_{y}} / d \bar{k}=\left(v_{2}(\bar{c})-v_{1}(\bar{l})\right) / \bar{k}$. It follows from equations (5), then, that $d \overline{W_{y}} / d \bar{k}=0$. Any deviation from the steady state capital stock would decrease welfare of the subsequent generations and, therefore, cannot be a Pareto-improvement.

We are now in a position to intuitively explain the contrast between Propositions 2.1 and 2.2. The first point to be noticed is that the interest rate is negative at steady state (i.e. $R<1$ ) in both cases. ${ }^{3}$ In view of Proposition 2.1, the fact that the interest rate is strictly negative allows one to find an allocation that Pareto-improves the welfare of agents, provided that it converges to a lower level of capital. This

\footnotetext{
${ }^{3}$ This was not made explicit in Reichlin [4]. However, following Reichlin's [4] notations, as $R=1 / a_{1}-1$ when $\delta=1$, the interest rate $r=1 / a_{1}-2$ is strictly negative whenever the conditions ensuring local indeterminacy and Hopf bifurcation are fulfilled, under Leontief technology, as they imply $1<1 / a_{1}<2$.
} 
is because the concavity of the production function under capital-labor substitution increases the marginal product of capital when capital goes down. Under Leontief technology, however, the marginal product of capital is constant (that is, $R-1+\delta=1 / a_{1}-2+\delta$ ) so that there is no way to generate higher consumption (when $1 / a_{1}>1$ ) by decreasing the capital stock, as $\bar{c}=\left(1 / a_{1}-\delta\right) \bar{k}$. By comparing Proposition 2.1, on the one hand, and both Proposition 2.2 and Reichlin's [4] appendix on the other, one realizes that the steady state is not Pareto-optimal when it is a sink or when it bifurcates, except under the assumption of Leontief technology. The most important implication is that the policies advocated by Reichlin [4, Section 3] and targeting the stationary allocation do not alleviate the associated welfare loss, except under the very special case of Leontief technology. As the empirical evidence suggests that substitution between factors exists up to some degree, it appears rather obvious that, apart from the fixed-coefficient case on the production side, the reversal Pareto-suboptimality property deceives the policy maker: should have Reichlin taken into account that, in his extension of the model to a CES technology, the steady-state was never Pareto-optimal, he would have concluded, as we do, that such stabilization policies would be not desirable. Therefore, if one considers the broader class of CES produc- 
tion functions (see Reichlin [4, Section 4, pp. 97-98]), the analysis carried out in Reichlin [4, Section 3] cannot be applied whenever capital-labor substitution is arbitrarily close, but different, from zero.

\section{References}

[1] Cazzavillan, G., Lloyd-Braga, T., Pintus, P., 1998. Multiple steady states and endogenous fluctuations with increasing returns to scale in production. Journal of Economic Theory 80, pp. 60-107.

[2] Cazzavillan, G., 2001. Indeterminacy and endogenous fluctuations with arbitrarily small externalities. Journal of Economic Theory 101 pp. $133-157$.

[3] Cazzavillan, G., Pintus, P., 2005. Endogenous business cycles and dynamic inefficiency. Forthcoming in the International Journal of Economic Theory.

[4] Reichlin, P., 1986. Equilibrium cycles in an overlapping generations economy with production. Journal of Economic Theory 40, pp. 89102. 